\title{
Assessment of Laryngeal Mask Airway in General Anesthesia during Thyroidectomy without Applying Miorelaxants
}

\author{
Aynur Isayeva \\ Scientific Surgery Centre Named after M. A. Topchubashov, Baku AZ1000, Azerbaijan
}

\begin{abstract}
The selective approach of anesthesia has a crucial role in the surgical treatment of thyroid diseases. Anesthesia during the insertion of a laryngeal mask airway is an alternative instead of endotracheal tube, hence this is a novel period in anesthesiology.
\end{abstract}

Key words: Laryngeal mask airway (LMA), thyroidectomy, adrenocorticotropic hormone (ACTH), Cortisol, bispectral index (BIS).

\section{Introduction}

The use of an endotraxeal tube in general anesthesia requires the establishment of deep sedation in the patient and the use of miorelaxants [1]. However, after tracheal intubation and extubation can occur some complications during and after surgery [2]. These complications include hyperdynamic response of the cardiovascular system to intubation and extubation, disruption of the corneal epithelium, and complications like as pharyngitis, laryngitis [3]. The frequency of such symptoms varies between $18-80 \%$, depending on the nature of the surgical intervention and the individual characteristics of the patient [4]. Providing adequate anesthesia with LMA in thyroidectomy and preventing possible complications is one of the pressing problems of modern anesthesiology [5-7].

\section{Materials and Methods}

The tests were conducted on 2 groups of patients: Group I-60 patients undergoing general anesthesia with the use of LMA and 20 patients undergoing anesthesia with generalized anesthesia with intubation of trachea. Patients younger than 50 years were predominant (60\%

Corresponding author: Aynur Isayeva, Ph.D., research fields: anesthesiology. in the main group and $57.5 \%$ in the control group). The number of women was 4.5 times more that of men in the group I and 4 times more in the control (II) group. All patients in both groups are included in ASA I. In group I, we provided the ventilation with a laryngeal mask while maintaining spontaneous breathing of the patient without the use of a relaxant, and in the control group, the mechanical ventilation was provided by the classical intubation of trachea in the intermittent positive pressure ventilation (IPPV) mode. We used $10.5 \pm 1.05$ seconds for using LMA and $9.5 \pm 4.0$ seconds for intubation of trachea; intubation was repeated in one patient. Twenty patients each had a comparative evaluation of the levels of cortisol, ACTH, and glucosa from the stress factors; we have designated the bispectral of the brain (BIS) to control the adequacy of general anesthesia in these patients.

\section{Results and Discussion}

During the using LMA pulse rate in one minute in group I was $72.0 \pm 2.0$ and was $16.3 \%$ less than the same indication for group II with tracheal intubation ( $p$ $<0.05)$. Arterial blood pressure indicators were similar: systolic blood pressure (BP) in the main group was 13.6\%, diastolic BP 9.5\%, and mean BP 7.3\% less than control group II. In all 2 groups, hemodynamics 
parameters were stable during all stages of anesthesia. In group II, extubation resulted in an increase in all indicators of hemodynamics: systolic BP increased by $13.6 \%$, diastolic BP increased by $13 \%$, and mean BP increased by $8.3 \%$; in this group, pulse rate in one minute was $9.4 \%$ higher than that of the main group during extubation. In the main group, there were no hyperdynamic changes in hemodynamic parameters in the removal of the laryngeal mask. In both groups, ventilation parameters were normal. In each of the 2 groups, the BIS index at all anesthesia periods was 45.5 \pm 3.6. In group II, the level of cortisol during intubation and extubation was slightly higher than that of the group I, but still did not exceed normal values. In each of the 2 groups, the results of ACTH in blood coincided with the results of cortisol.

\section{Conclusions}

During the general anesthesia on thyroidectomy the use of LMA and endotracheal tube with propofol and isoflurane, the parameters of ventilation, arterial blood gas (ABG), BP, BIS monitor results were normal. In the initial postoperative period of anesthesia with the use of LMA compared with endotrachal anesthesia, the Aldert scale was maximized, blood cortisol and ACTH levels were reported at lower levels (25 $\pm 1.5 \%)$, decreased postoperative recovery period (35 $\pm 5 \%$ ), postoperative discomfort in the throat. Patients with non-toxic goiter who do not have any other disease, laryngeal mask airway can be obtained with adequate anesthesia, and this method can be considered an alternative to general anesthesia with tracheal intubation.

\section{References}

[1] Frerk, C., Mitchell, V. S., McNarry, A. F., Mendonca, C., Bhagrath, R., Patel, A., et al. 2015. "Difficult Airway Society 2015 Guidelines for Management of Unanticipated Difficult Intubation in Adults.” $\mathrm{Br} \mathrm{J}$ Anaesth 115: 827-48.

[2] Mehanna, R., Hennessy, A., Mannion, S., O’Leary, G., and Sheahan, P. 2015. "Effect of Endotracheal Tube Size on Vocal Outcomes after Thyroidectomy: A Randomized Clinical Trial.” JAMA Otolaryngol Head Neck Surg. 141 (8): 690-5

[3] Kashif, S., and Gulrez, A. 2017. "Anaesthetic Concerns of Surgical Emphysema Following Thyroidectomy.” J Coll Physicians Surg Pak 27 (3): S4-5.

[4] Chun, B. J., Bae, J. S., Lee, S. H., Joo, J., Kim, E. S., and Sun, D. 2015. "Prospective Randomized Controlled Trial of the Laryngeal Mask Airway versus the Endotracheal Intubation in the Thyroid Surgery: Evaluation of Postoperative Voice, and Laryngopharyngeal Symptom.” $\begin{array}{lllll}\text { World } J & \text { Surg } & 39 & \text { (7): } & \text { 1713-20. }\end{array}$ doi:10.1007/s00268-015-2995-7.

[5] Popescu, R. 2014. "Neuromonitoring the Laryngeal Nerve during Thyroidectomy-Literature Review and Initial Experience." Romanian Journal of Rhinology 4 (13): 15-20.

[6] Jarineshin, H., Kashani, S., Vatankhah, M., Abdulahzade Baghaee, A., Sattari, S., and Fekrat, F. 2015. "Better Hemodynamic Profile of Laryngeal Mask Airway Insertion Compared to Laryngoscopy and Tracheal Intubation.” Iran Red Crescent Med J. 17 (8): e28615.

[7] Kendall, M. C. 2019. "Laryngeal Mask Airway as a Rescue Device for Failed Endotracheal Intubation.” Eur $J$ Emerg Med. 26 (1): 73. 